\title{
A complete optimization-loop, including feeders, of antenna systems based on reflectors
}

\author{
D. Delgado, J. Teniente, C. del-Río, L. de-Haro ${ }^{1}$, J.A. Martinez ${ }^{2}$ and A. G.Pino ${ }^{2}$ \\ Antenna Group, Universidad Pública de Navarra, \\ Campus Arrosadía s/n, E-31006 Pamplona, Spain. \\ ( $\left.{ }^{1}\right)$ SSR Depart. Universidad Politécnica de Madrid, E-28040, Madrid, Spain \\ $\left(^{2}\right)$ Antenna Group, Universidad de Vigo, E-36310 Vigo, Spain
}

In the design process of a complete antenna system based on reflectors, the feed real performance is usually included once the reflectors have been optimized with an ideal feed. In this paper, the proposal is to include the feed inside the optimization loop of the whole system, by means of a parametric definition of the feeder. To perform the optimization, the parametric feed will be considered as an additional surface, which receives some desired electric field that should be analyzed to properly modify the feed definition parameters in order to reduce the difference between the desired and the new generated field distribution over a planar surface nearby the focal point of the system. At the end of the optimization process, all the elements of the whole antenna system, reflectors and feed, will be totally defined and perfectly coupled.

\section{Introduction}

There are different research groups and commercial codes [1-3] available to analyze and optimize the shape of the reflector surfaces and feed positions of an antenna system. All of them use as an input parameter the feed type to be used in the optimization procedure. Usually, some ideal Gaussian or cos- $q$ distributions, or the real data of a particular feed, are assumed to be unchanged during all the shaping or the optimization process of the surfaces of the different reflectors included in the antenna system.

The proposal presented in this paper includes the feed inside the optimization loop where a gain level referred to a goal per test point is maximized. To do this, we will consider the feed as an additional surface to be optimized, but instead to act directly by changing the surface itself, we will try to identify the main features of the desired field distribution and to modify the feed parameters in order to minimize the difference between the desired field distribution and the finally obtained one after the changes performed in the feed.

We have chosen the Gaussian Profiled Horn Antennas (GPHA) [4,5] to define the parametric feed (figure 1), but many others could be used. The only restriction for selecting another antenna type will be the capability of modifying analytically the radiation performance by means of tuning a set of geometrical parameters. One of the clear advantages of the GPHA's, is the appropriate combination of return loss bandwidth, side-lobe and cross-polar levels, stability of the phase center, etc., that these kind of antennas exhibit naturally.

A plane, referred as the Feed Optimization Plane (FOP), is defined nearby the focal region, where the feed is planed to be placed. Considering the FOP as an additional 
surface to be optimized, the desired field distribution will be obtained at the FOP once per iteration step of the optimization procedure. This desired field should be analyzed and recognized as something similar to be launched by a horn antenna. Afterwards, the geometrical parameters of the antenna should be modified in order to reduce the difference between the desired and the obtained field distribution at the FOP.

\section{Working principle}

Initially, as usual, some analytical approach should be performed in order to establish the number of reflectors as well as their sizes and spatial positions to be used in the system.

One of the most important feed parameters to be defined is the directivity. The directivity can be specified by the illumination at certain azimuthal or elevation angles or directly by the far-field directivity or gain. If an analytical model is used, other possibilities could also be considered as, for instance, the parameter $q$ of a $\cos -q$ field distribution.

In our case, because of the particular shape of the selected feed, the GPHA, the type of possible illuminations offered by the feed will be very similar to a gaussian field distribution. The relation between the excited Gaussian beam and the geometrical parameters of the horn is well known [5]. Therefore, the parameter to be extracted from the desired field distribution will be the position and the size of the equivalent beamwaist.

The electric field at the FOP will be represented in amplitude and phase. We assume that the field distribution across the FOP, in the stationary state is similar to a gaussian field distribution launched by the feed. Thus, despite we could obtain quite distorted field distributions in the first iterations of the optimization procedure, we always will be launching a pure gaussian beam, with the parameters that fit as better as possible those extracted from the plane. In figure 2 the goal and the obtained parameters are compared showing that the agreement is really high.

In order to obtain the beam parameters, a parametric correlation integral (comparing the desired fields with gaussian field distributions with different parameters) could be considered, but this would be a long-time consuming procedure to be included in the optimization loop, so a simpler method is performed.

Thus, in order to estimate the position of the phase center, $z_{0}$, it is enough to evaluate, in a set of points of the FOP, the equivalent curvature radius, $R\left(z_{p}\right)$, through the phase difference of these points and the central point. The equation defining the relation of the curvature radius of a Gaussian field distribution is:

$$
R(z)=\left(z-z_{0}\right)\left[1+\left(\frac{k w_{0}^{2}}{2\left(z-z_{0}\right)}\right)^{2}\right]
$$

being $w_{0}$ the beam-waist, this is, the minimum transversal beam-width and $z_{0}$ its position. If the field distribution matches perfectly with a gaussian distribution, all the evaluations will provide the same curvature radius, else the selected value will be the one which provides a maximum match. 
In equation 1 , two unknowns appear, $w_{0}$ and $z_{0}$, so another equation is needed in order to be able to solve the problem. So, we will use the beam-width value, $w\left(z_{p}\right)$, and it could be obtained comparing the sampled amplitude distribution with some normalized threshold $(1 / e)$; and modeling the obtained matrix as a circle of radius $w\left(z_{p}\right)$. As in the case of the curvature radius, the beam-width value is a function of $w_{0}$ and $z_{0}$,

$$
w(z)=w_{0} \sqrt{1+\left(\frac{2\left(z-z_{0}\right)}{k w_{0}^{2}}\right)^{2}}
$$

Combining these two values obtained, the curvature radius and the beam width on the plane, $R\left(z_{p}\right)$ and $w\left(z_{p}\right)$, the position and the size of the beam-waist, $w_{0}$ and $z_{0}$, can be extracted.

At this point we will have all the data to completely define a GPHA. In fact, only the beam-waist value is necessary, because the final position of this beam will be very dependant of the particular horn profile. So, as a result of the horn definition, the relative position of the phase center inside the horn will be obtained, so we could use that information to reallocate the horn in relation with the FOP.

A natural limitation of the achievable directivities using a corrugated feed is determined to values between the range of $18-27 \mathrm{~dB}$, which corresponds with beam-waist range of 0.7-2.4, approximately. For higher directivities, the corrugated horn antenna becomes too large; and for lower directivities other horn technologies should be selected for more compact designs.

At the end of the whole optimization procedure, we will obtain an optimum horn antenna profile, strongly coupled with the rest of the antenna system.

\section{Conclusion}

In this paper, a novel procedure to include the feed parameters of a Gaussian Profile Corrugated Horn Antenna into the optimization procedure of a reflector antenna system has been proposed.

\section{References:}

[1] http://www.gr.ssr.upm.es/sabor.htm

[2] http://ticra.wpsdemo.com/script/site/page.asp?artid=33\&cat id $=30$

[3] J. A. Martínez, A. G. Pino, M. Arias, O. Rubiños, "ANDERA: A Software for the analysis and design of multi-fed and shaped reflector antennas", AP-S International Symposium and USNC/URSI National Radio Science Meeting, IEEE, Monterey CA. 2004.

[4] Teniente, J., Gonzalo, R. and Del Río, C., "Conical versus Gaussian Profiled Horn Antennas", 2002 IEEE AP-S International Symposium, San Antonio, July 2002.

[5] Teniente,J., "Modern Corrugated Horn Antennas", Ph. D. manuscript published in Universidad Pública de Navarra, Pamplona (Spain), July 2003 


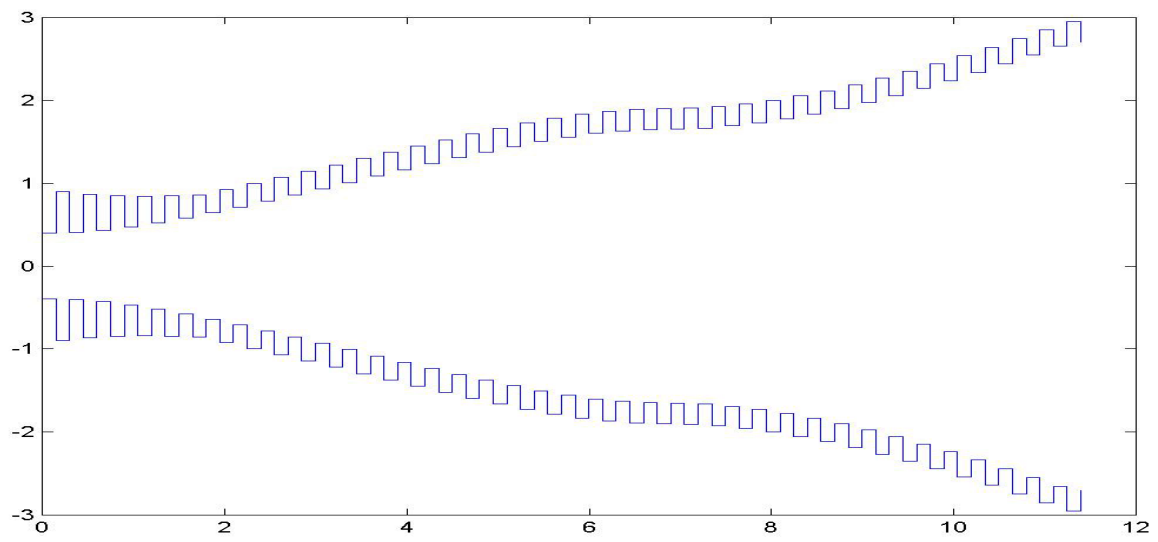

Figure 1.- The GPHA parametric type used in the optimization loop is shown. All dimensions expressed in wavelengths.

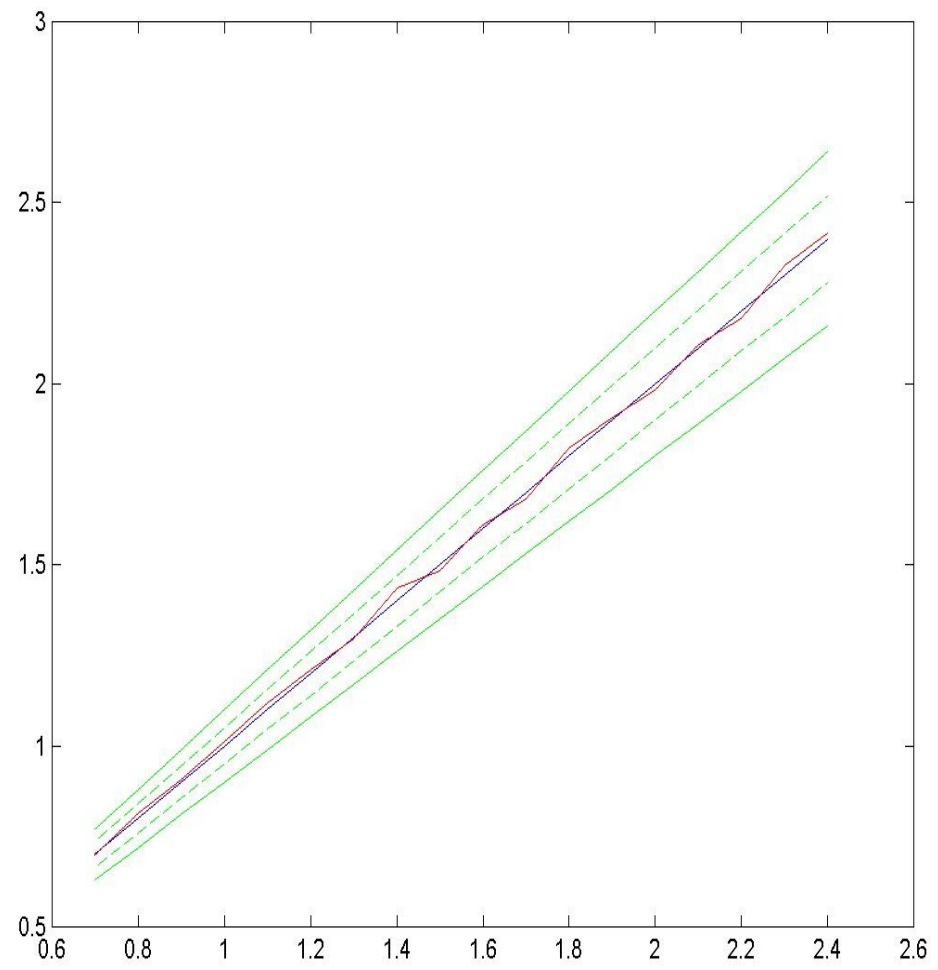

Figure 2.- Comparison between the desired (horizontal axis) and the obtained (vertical axis) beam-waist by modifying analytically the geometrical parameters of a GPHA. The dashed line fits the 5\% error and the continuous line the $10 \%$ error. 